\title{
A large annular scaly plaque
}

Gwyneth N Wong, Jane Li,

Matheen Mohamed, Richard Williams, Alvin Chong

\section{CASE}

A Caucasian man aged 60 years presented with a six-month history of an annular, red, scaly plaque on his posterior thigh (Figure 1a). The plaque had started as a small papule, which then slowly expanded to $>10 \mathrm{~cm}$ in diameter. He was systemically well but had a past history of alcohol dependence and liver cirrhosis.

\section{Question 1}

What conditions commonly present as annular scaly plaques?

\section{Question 2}

What additional history would be useful for refining the differential diagnosis?

\section{Question 3}

What initial investigations would be appropriate?



\section{Answer 1}

Possible differential diagnoses include:

- tinea corporis

- psoriasis

- nummular eczema

- erythema annulare centrifugum

- subacute cutaneous lupus erythematous

- mycosis fungoides

- secondary syphilis.

\section{Answer 2}

Useful information to elucidate on history includes:

- any previous rashes

- comorbidities such as diabetes or immunosuppression that could predispose to infections

- personal or family history of autoimmune disease and neoplasia

- sexual history and any prior history of sexually transmissible infections (STIs).

\section{Answer 3}

Punch biopsy of the lesion would be appropriate. A basic blood panel and serology for STIs could be considered if the patient appeared high risk.

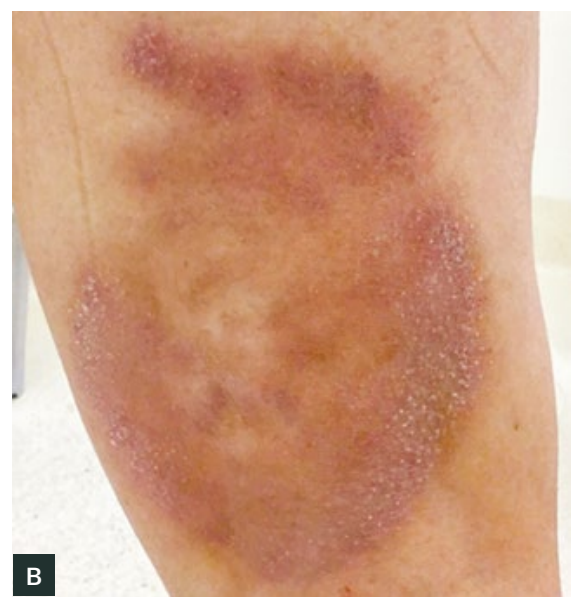

Figure 1. An annular erythematous scaly plaque with a width of $10 \mathrm{~cm}$ on the patient's posterior thigh $A$. The plaque prior to treatment; $B$. The resolving plaque seven weeks after treatment

\section{CASE CONTINUED}

On further questioning, the patient reported that his last sexual contact was with a local female in Thailand 12 months prior. He recalled a painless penile ulcer developing about three months later, which self-resolved. A punch biopsy was performed, revealing spongiosis with a superficial and deep perivascular lymphohistiocytic infiltrate and numerous plasma cells (Figure 2). ${ }^{1}$ Syphilis serology was subsequently ordered. Enzyme immunoassay was positive for Treponema pallidum and rapid plasma reagin (RPR) test showed a high titre of $>$ 512. T. pallidum particle agglutination assay was also positive, consistent with active syphilis. Screening for human immunodeficiency virus, Chlamydia trachomatis and Neisseria gonorrhea proved negative.

\section{Question 4}

What feature on skin biopsy is suggestive of this condition? Why is serological testing required for definitive diagnosis?

\section{Question 5}

What is the appropriate treatment and follow-up for this condition?

\section{Answer 4}

The presence of numerous plasma cells on skin biopsy should alert the clinician to the possibility of syphilis. Clinicians should proceed to order syphilis serology, which is the mainstay of laboratory diagnosis, given the inability to culture the causative organism. ${ }^{2}$ Spirochaetes may also be demonstrable on immunohistochemical or Warthin-Starry silver stains. ${ }^{2}$

\section{Answer 5}

Syphilis remains sensitive to penicillin, so a single dose of intramuscular benzathine penicillin remains the standard first-line treatment. ${ }^{2}$ Follow-up 
serological testing with repeat RPR at three, six and 12 months after treatment is recommended to monitor response to treatment, as there is an absence of a microbiologic test for cure. ${ }^{2} \mathrm{~A}$ fourfold or greater reduction in RPR indicates adequate response to treatment. Syphilis is a notifiable disease in Australia and contact tracing is advised.

\section{CASE CONTINUED} infectious diseases unit and responded well to a single dose of $1.8 \mathrm{~g}$ intramuscular benzathine penicillin. There was clinical improvement with partial resolution of the plaque at seven weeks after treatment (Figure 1B), followed by full resolution by six months after treatment. A four-fold reduction in RPR titre was also observed.

\section{Discussion}

Syphilis is an STI caused by the spirochaete T.pallidum. The global prevalence and incidence of syphilis remain high. ${ }^{3}$ In Australia, the incidence of syphilis has been increasing, particularly among high-risk groups such as men who have sex with men. ${ }^{4}$ This case highlights the difficulty in diagnosing syphilis given its protean clinical manifestations. Primary syphilis is characterised by a chancre - a painless and often indurated ulcer at the site of inoculation (most

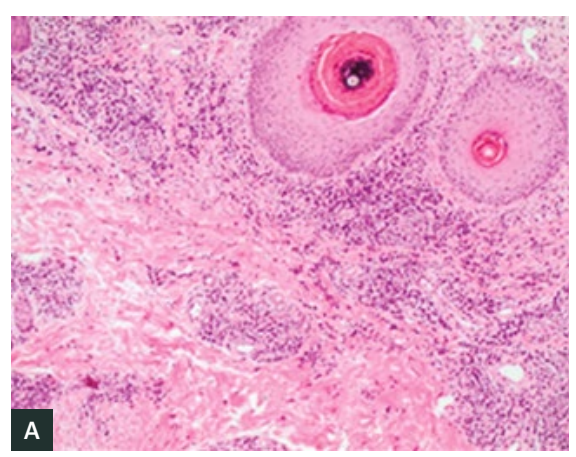

The patient was referred to the

commonly genital, anal or orolabial). By contrast, secondary syphilis generally presents as a polymorphic rash with lymphadenopathy and other systemic manifestations. An asymptomatic latent period then follows if the disease is not treated, leading to a tertiary stage that may involve severe cardiovascular and neurologic sequelae and gummatous changes to any organ system. ${ }^{2}$

Previous studies have reported that up to $29.6 \%$ of cases of secondary syphilis have cutaneous manifestations with atypical morphology. ${ }^{5}$ Atypical cutaneous manifestations of secondary syphilis include nodular, annular, pustular, framboesiform, lues maligna and photodistributed papulosquamous eruptions. ${ }^{6}$ Differentials for annular secondary syphilis, depending on anatomic site and clinical scenario, include dermatophytosis, erythema annulare centrifugum, lichen planus, subacute cutaneous lupus erythematosus, sarcoidosis, atypical mycobacterial infection and granuloma annulare..$^{7-9}$

\section{Key points}

- Syphilis is a 'great mimicker' and should not be overlooked as a differential diagnosis of erythematous scaly rashes, especially as its global prevalence and incidence remain high. The incidence in high-risk populations in Australia has recently been increasing.

- The presence of numerous plasma cells on skin biopsies should signal the

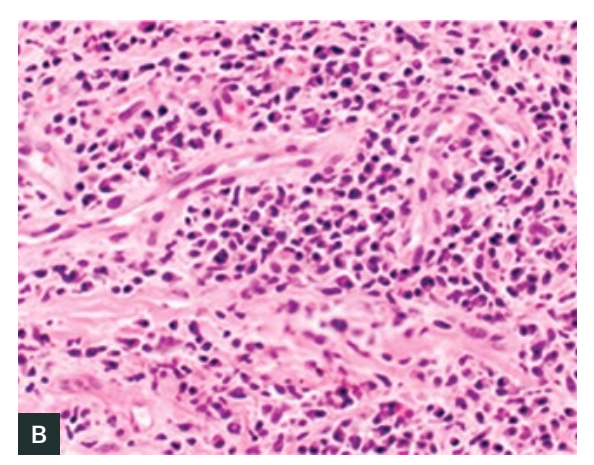

Figure 2. Punch biopsy specimen from posterior thigh prior to treatment

A. Vascular proliferation and perivascular inflammatory infiltrate (H\&E, x100); B. The majority of inflammatory cells were plasma cells (H\&E, x400) clinician to the possibility of syphilis and the need to order syphilis serology.

- Early diagnosis of syphilis is vital as it is easily treated in its early stages with a single dose of benzathine penicillin. Timely and effective treatment of early syphilis is important to prevent onward transmission and progression to tertiary syphilis.

\section{Authors}

Gwyneth N Wong MBBS, BMedSci, Medical Officer, Department of Dermatology, St Vincent's Hospital, Melbourne, Vic. g.natalie.wong@gmail.com Jane Li MBBS, Dermatology registrar, Department of Dermatology, St Vincent's Hospital, Melbourne, Vic Matheen Mohamed MBBS, FACD, MPH, Consultant Dermatologist, Department of Dermatology, St Vincent's Hospital, Melbourne, Vic

Richard Williams MBBS, FACP, Consultant Pathologist, Department of Anatomical Pathology, St Vincent's Hospital, Melbourne, Vic

Alvin Chong MBBS, FACD, Consultant Dermatologist, Department of Dermatology, St Vincent's Hospital, Melbourne, Vic Competing interests: None.

Funding: None.

Provenance and peer review: Not commissioned, externally peer reviewed.

\section{References}

1. Wong GN, Li J, Williams A, Mohamed M, Chong $\mathrm{AH}$. Case report: Secondary syphilis masquerading as tinea corporis. Australas J Dermatol 2017;58(Suppl 1):101.

2. Singh AE, Romanowski B. Syphilis: Review with emphasis on clinical, epidemiologic, and some biologic features. Clin Microbiol Rev 1999;12(2):187-209.

3. Newman L, Rowley J, Vander Hoorn S, et al. Globa estimates of the prevalence and incidence of four curable sexually transmitted infections in 2012 based on systematic review and global reporting. PLoS One 2015;10(12):e0143304. doi: 10.1371/ journal.pone.0143304.

4. Jin F, Prestage GP, Kippax SC, et al. Epidemic syphilis among homosexually active men in Sydney. Med J Aust 2005;183(4):179-83.

5. Hira SK, Patel JS, Bhat SG, Chilikima K, Mooney N. Clinical manifestations of secondary syphilis. Int J Dermatol 1987;26(2):103-07. doi: 10.1111/j.13654362.1987.tb00532.x.

6. Balagula Y, Mattei PL, Wisco OJ, Erdag G, Chien AL. The great imitator revisited: The spectrum of atypical cutaneous manifestations of secondary syphilis. Int J Dermatol 2014;53(12):1434-41. doi: 10.1111/ijd.12518.

7. Sarojini PA, Dharmaratnam AD, Pavithran $K$, Gangadharan C. Concentric rings simulating tinea imbricata in secondary syphilis. A case report. Br J Vener Dis 1980;56(5):302-03.

8. Pournaras CC, Masouye I, Piletta P, Piguet V, Saurat JH, French LE. Extensive annular verrucous late secondary syphilis. Br J Dermatol 2005;152(6):1343-45. doi: 10.1111/j.13652133.2005.06546.x.

9. Cotterman C, Eckert L, Ackerman L. Syphilis mimicking tinea imbricata and erythema annulare centrifugum in an immunocompromised patient. J Am Acad Dermatol 2009;61(1):165-67. doi: 10.1016/j.jaad.2008.10.027. 